\title{
A Case of Ischiofemoral Impingement Syndrome as a Differential Diagnosis of Ankylosing Spondylitis
}

\author{
Minkoo Kang ${ }^{1}$, So-Young Bang ${ }^{2}$, Jeong Ah Ryu ${ }^{3}$, Seungjun Gim, Eun-Sik Park ${ }^{1}$, Hyeyoung Lee ${ }^{1}$, Hye-Soon Lee ${ }^{2}$ \\ ${ }^{1}$ Department of Internal Medicine, Hanyang University College of Medicine, Seoul, ${ }^{2}$ Division of Rheumatology, Department of Internal \\ Medicine, ${ }^{3}$ Department of Radiology, Hanyang University Guri Hospital, Guri, Korea
}

\begin{abstract}
Ischiofemoral impingement (IFI) syndrome is an uncommon cause of gluteal and hip pain. We report on a case of a 20-year-old man who presented with chronic gluteal and hip pain with low back pain without a history of trauma or surgery. He was misdiagnosed with ankylosing spondylitis (AS) at another clinic. The patient was finally diagnosed with IFI syndrome according to pelvic magnetic resonance imaging findings at our hospital. After two weeks of medical and physical treatment, his pain showed gradual improvement. Because IFI syndrome is rarely reported in male patients, it might be misdiagnosed as AS. Therefore, IFI syndrome should be considered as a differential diagnosis of AS, particularly in young male patients with atypical pain characteristics. (J Rheum Dis 2016;23:179-182)
\end{abstract}

Key Words. Ischiofemoral impingement syndrome, Ankylosing spondylitis

\section{INTRODUCTION}

Ischiofemoral impingement (IFI) is defined by a decreased space between the lesser trochanter and the ischium with entrapment of the quadratus femoris muscle. Since the first case was described in 1977 [1], many cases have been reported, most of which have been in women with a history of trauma or surgery. Even though clinical and radiographic characteristics of IFI syndrome are now well-defined, it can present atypically with vague symptoms of gluteal and lower back pain, making proper diagnosis difficult or delayed. Here, we report a case of IFI syndrome in a young man who had been misdiagnosed as having ankylosing spondylitis (AS).

\section{CASE REPORT}

A 20-year-old man visited our clinic to get a second opinion regarding a previous diagnosis of AS at a military hospital. He presented with both gluteal and hip pain with low back pain for the previous six months. His pain persisted throughout the day and while sleeping at night. The pain was dull, increased in intensity when walking, and did not radiate. He had no history of trauma or surgery. When the symptoms began, he had been playing tennis frequently.

According to a medical certificate by the referring doctor, he was diagnosed with AS based on his chronic gluteal pain with low back pain as well as findings of sacroiliitis on plain radiographs of the right sacroiliac (SI) joint.

On physical examination, results of the modified Schober test, the occiput-to-wall distance test and the chest expansion test were within normal limits. Full and symmetric range of motion of both hips was preserved. Buttock and hip pain was elicited with extension, adduction and external rotation of both hip joints. Tenderness was present mid-buttock but not at the SI joint. There was no lower extremity atrophy or deformity. Result of a muscle strength test and sensory examination of bilateral lower extremities showed no abnormal findings. Deep ten-

\footnotetext{
Received : July 8, 2015, Revised : (1st) August 11, 2015, (2nd) August 26, 2015, Accepted : August 27, 2015

Corresponding to : Hye-Soon Lee, Division of Rheumatology, Department of Internal Medicine, Hanyang University Guri Hospital, 153 Gyeongchun-ro, Guri 11923, Korea. E-mail : Ihsberon@hanyang.ac.kr 
don reflexes of bilateral knees and ankles were normal. Results of straight leg raising test were negative for both sides.

Laboratory findings were normal, including a white blood cell count of $9,900 / \mathrm{mm}^{3}$, hemoglobin level of 15.5 $\mathrm{g} / \mathrm{dL}$, platelet count of $298,000 / \mathrm{mm}^{3}$, C-reactive protein concentration of $0.25 \mathrm{mg} / \mathrm{dL}$ (reference range 0 to 0.8 $\mathrm{mg} / \mathrm{dL}$ ), and erythrocyte sedimentation rate of $5 \mathrm{~mm} / \mathrm{h}$ (reference range 0 to $10 \mathrm{~mm} / \mathrm{h}$ ). Other laboratory findings, including blood chemistry and coagulation tests also were within normal limits. Human leukocyte antigen-B27, rheumatoid factor, anti-nuclear antibody, and anti-cyclic citrullinated peptide antibody were all negative.

We reviewed plain radiographs of the pelvis and lumbar spine from the referring hospital as well as new radiographs from our hospital, in which only suspicious mild sclerotic lesions of the right SI joint were observed (Figure 1). To confirm the early changes of the SI joint in AS, we performed magnetic resonance imaging (MRI) of the pelvis.

Axial T2-weighted fat-suppressed MRI showed no evidence of sacroiliitis, with no sclerosis, erosions, alternations of joint width, or ankylosis of the SI joint, or surrounding bone marrow edema (Figure 2A). However, imaging did reveal bilateral narrowing of the ischiofemoral space (IFS) with increased signal intensity of the quadratus femoris muscles, suggesting diffuse edema (Figure 2B). The length of the IFS was $12.7 \mathrm{~mm}$ on the right side and $11.5 \mathrm{~mm}$ on the left side, while the length of the quadratus femoris space (QFS) was $7.7 \mathrm{~mm}$ on the right side and $9.8 \mathrm{~mm}$ on the left side, all of which are compatible with bilateral IFI. No cystic changes within the is-

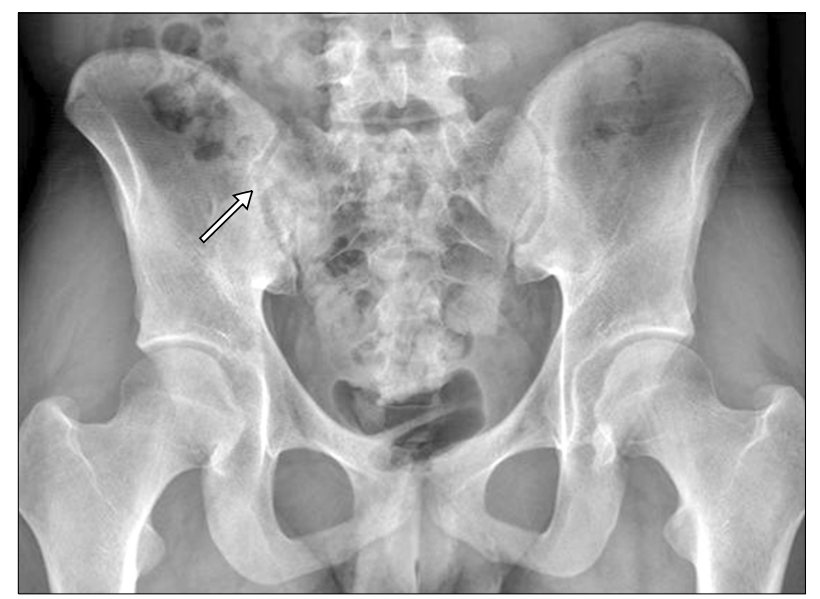

Figure 1. Plain radiograph of the pelvis, showing suspicious mild sclerotic lesions of the right sacroiliac joint (arrow). chium or abnormalities of the hamstring tendons were observed. There were no findings of degenerative arthritis, femoroacetabular impingement, osteonecrosis, or posttraumatic deformity, which are possible causes of pain.

After IFI syndrome was diagnosed, the patient was treated with oral nonsteroidal anti-inflammatory drugs, analgesics, physical therapy and exercise education. After two weeks of treatment, his pain intensity improved from a visual analog scale of $7 \sim 8 / 10$ to $1 \sim 2 / 10$.

\section{DISCUSSION}

AS is a chronic inflammatory disease of the axial skeleton, that most commonly affects men younger than 40 years of age. It mainly affects the joints in the spine and the SI joint in the pelvis. The most common symptoms of AS are chronic dull pain in the lower back or gluteal region and stiffness of the lower back, which often is severe in the morning or at rest but improves with physical activity. However, because many patients experience varying degrees of pain regardless of activity, accurate diagnosis can be difficult sometimes. Therefore, cases with atypical chronic lower back or gluteal pain, especially in young men require thoughtful consideration of differ-

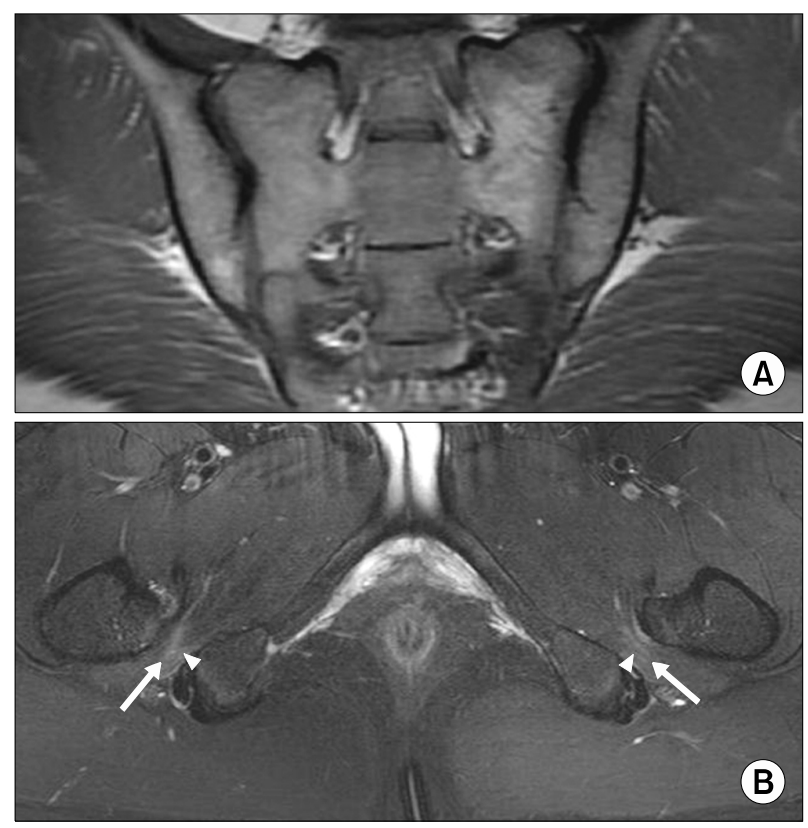

Figure 2. (A) T1-weighted magnetic resonance imaging (MRI), showing no definite findings of sacroiliitis. (B) T2-weighted fat-suppressed MRI, revealing bilateral narrowing of the ischiofemoral space (arrows) with increased signal intensity of the quadratus femoris muscles (arrowheads). 
ential diagnosis to confirm or exclude AS.

Here, we presented a case of young man with IFI syndrome who had been previously misdiagnosed as having AS, emphasizing that IFI syndrome should be considered as a differential diagnosis of AS.

IFI syndrome was first described in a report by Johnson [1] in 1977, in which three patients developed IFI syndrome after hip surgery including total hip arthroplasty and proximal femoral osteotomy. Recently, several cases of IFI syndrome with no association to previous surgery or trauma have been reported and managed with a nonsurgical approach [2-4]. In prior reports, IFI syndrome was largely described in adult women [5]. To our knowledge, only a single case of IFI syndrome has been published, describing male patient with no history of surgery or trauma [4]. However, this patient failed treatment with medication or physical rehabilitation unlike in our case.

While both AS and IFI syndrome can present with gluteal and hip pain, the characteristics of the pain might be different. Our case showed that aggravation of pain with exercise is compatible with IFI syndrome rather than AS. However, the patient also had pain at rest which made proper diagnosis difficult, resulting in misdiagnosis as AS. In addition, the lower back pain in the current case also was a confusing symptom of AS, which might have originated from the muscles around the lumbosacral region combined with IFI syndrome. As suggested in many prior reports of IFI syndrome, recent exercise might have been a triggering factor $[3,6,7]$.

Diagnosis of IFI syndrome can be established with both clinical and radiologic findings. MRI is the gold standard diagnostic test and should demonstrate narrowing of the IFS and QFS as well as quadratus femoris muscle abnormalities. The IFS is defined as the smallest distance between the lateral cortex of the ischial tuberosity and the medial cortex of the lesser trochanter, while the QFS is the smallest space for passage of the quadratus femoris muscle delimited by the superolateral surface of the hamstring tendons and the posteromedial surface of the iliopsoas tendon or lesser trochanter [8]. The normal distance between the lesser trochanter and the ischial tuberosity is approximately $20 \mathrm{~mm}$ with the hip in adduction, external rotation and extension [8]. In patients with IFI, this space is significantly narrower. Based on a prior meta-analysis, optimally balanced specificity and sensitivity profiles resulted in thresholds of $15 \mathrm{~mm}$ and $10 \mathrm{~mm}$ for IFS and QFS narrowing, respectively [5]. An abnormal relationship between the lesser trochanter and the ischium can lead to injury of the soft tissues, such as the quadratus femoris muscle, potentially resulting in hip pain. Our patient's IFS and QFS measurements were consistent with IFI.

Interpretation of radiographs of the SI joint might contribute to a misdiagnosis of AS, as in our case. While this patient's diagnosis of AS was based on findings of sacroiliitis on plain radiographs at the referring hospital, an expert radiologist at our hospital interpreted the film as a suspicious mild sclerotic region of the right SI joint. Although plain radiography is essential for the diagnosis of sacroiliitis, it has several limitations, including low sensitivity and substantial extent of intra- and interobserver variability [9]. Therefore, interpretation using simple radiographs alone should be cautious regardless of changes in the SI joint [10]. MRI has higher sensitivity and specificity for the diagnosis of sacroiliitis, especially in the early stages [11]. A prior study comparing MRI to conventional radiography of the SI joint showed that approximately $16 \%$ of the findings that led to a diagnosis of sacroiliitis with conventional radiography were not observed on MRI [12]. Therefore, MRI evaluation of the SI joint is probably necessary to confirm AS, especially in cases with an atypical clinical presentation and without definite sacroiliitis on simple radiographs. Moreover, MRI can provide important clues that can exclude AS and improve the differential diagnosis to include IFI syndrome, as in our case. Therefore, when considering the various causes of lower back and gluteal pain, MRI should be used to scan the entire pelvis from the upper SI joint to the lower pelvis including the IFS.

\section{SUMMARY}

Although IFI syndrome is not a well-known source of gluteal and hip pain, it should be considered as a differential diagnosis of AS, especially in young men with atypical pain characteristics. Pelvic MRI can assist in the proper diagnosis of IFI syndrome.

\section{CONFLICT OF INTEREST}

No potential conflict of interest relevant to this article was reported.

\section{REFERENCES}

1. Johnson KA. Impingement of the lesser trochanter on the ischial ramus after total hip arthroplasty. Report of three 
cases. J Bone Joint Surg Am 1977;59:268-9.

2. Patti JW, Ouellette H, Bredella MA, Torriani M. Impingement of lesser trochanter on ischium as a potential cause for hip pain. Skeletal Radiol 2008;37:939-41.

3. López-Sánchez MC, Armesto Pérez V, Montero Furelos LÁ, Vázquez-Rodríguez TR, Calvo Arrojo G, Díaz Román TM. Ischiofemoral impingement: hip pain of infrequent cause. Reumatol Clin 2013;9:186-7.

4. Kim WJ, Shin HY, Koo GH, Park HG, Ha YC, Park YH. Ultrasound-guided prolotherapy with polydeoxyribonucleotide sodium in ischiofemoral impingement syndrome. Pain Pract 2014;14:649-55.

5. Singer AD, Subhawong TK, Jose J, Tresley J, Clifford PD. Ischiofemoral impingement syndrome: a meta-analysis. Skeletal Radiol 2015;44:831-7.

6. Safran M, Ryu J. Ischiofemoral impingement of the hip: a novel approach to treatment. Knee Surg Sports Traumatol Arthrosc 2014;22:781-5.

7. Papavasiliou A, Siatras T, Bintoudi A, Milosis D, Lallas V, Sykaras E, et al. The gymnasts' hip and groin: a magnetic resonance imaging study in asymptomatic elite athletes. Skeletal Radiol 2014;43:1071-7.
8. Torriani M, Souto SC, Thomas BJ, Ouellette H, Bredella MA. Ischiofemoral impingement syndrome: an entity with hip pain and abnormalities of the quadratus femoris muscle. AJR Am J Roentgenol 2009;193:186-90.

9. Yazici H, Turunç M, Ozdoğan H, Yurdakul S, Akinci A, Barnes CG. Observer variation in grading sacroiliac radiographs might be a cause of 'sacroiliitis' reported in certain disease states. Ann Rheum Dis 1987;46:139-45.

10. Cohen AS, McNeill JM, Calkins E, Sharp JT, Schubart A. The "normal" sacroiliac joint. Analysis of 88 sacroiliac roentgenograms. Am J Roentgenol Radium Ther Nucl Med 1967; 100:559-63.

11. Oostveen J, Prevo R, den Boer J, van de Laar M. Early detection of sacroiliitis on magnetic resonance imaging and subsequent development of sacroiliitis on plain radiography. A prospective, longitudinal study. J Rheumatol 1999;26:1953-8.

12. Poddubnyy D, Gaydukova I, Hermann KG, Song IH, Haibel $\mathrm{H}$, Braun J, et al. Magnetic resonance imaging compared to conventional radiographs for detection of chronic structural changes in sacroiliac joints in axial spondyloarthritis. J Rheumatol 2013;40:1557-65. 